\title{
Search for Magnetic Monopoles Trapped in Matter
}

\author{
Hunmoo Jeon and Michael J. Longo \\ University of Michigan, Department of Physics, Ann Arbor, MI 48109 \\ (to be published in Physical Review Letters)
}

(June, 1995)

\begin{abstract}
There have been many searches for magnetic monopoles in flight, but few for monopoles in matter. We have searched for magnetic monopoles in meteorites, schists, ferromanganese nodules, iron ores and other materials. The detector was a superconducting induction coil connected to a SQUID (Superconducting Quantum Interference Device) with a room temperature bore $15 \mathrm{~cm}$ in diameter. We tested a total of more than $331 \mathrm{~kg}$ of material including $112 \mathrm{~kg}$ of meteorites. We found no monopole and conclude the overall monopole/nucleon ratio in the samples is $<1.2 \times 10^{-29}$ with a $90 \%$ confidence level.
\end{abstract}

\section{BACKGROUND}

In 1931 Dirac [1] suggested that the existence of magnetic monopoles can explain the quantization of electric charge. Dirac showed that a monopole must carry a magnetic charge which is an integer multiple of $g_{D}=68.5$ e in CGS units. After many unsuccessful searches for monopoles, interest gradually waned until the Grand Unified Theory (GUT) magnetic monopole was introduced by t' Hooft [2] and independently by Polyakov [3] in 1974. They found that there is a solution with magnetic monopoles in any unified gauge theory which has an unbroken U(1) factor embedded in it. Since the U(1) factor is essential for describing electromagnetism, virtually all unified theories predict the existence of magnetic monopoles. The most notable property of the GUT monopoles is their extreme mass. In all GUTs the monopole mass $\left(m_{M}\right)$ is of the order $\sim 4 \pi m_{X} / e^{2}$ where $X$ is the mass of the superheavy vector boson, which determines the unification energy scale [4]. For the SU(5) GUT, $m_{X}$ is $\sim 10^{14} \mathrm{GeV}$ which implies $m_{M} \approx 10^{16} \mathrm{GeV}(\sim 0.01 \mu \mathrm{g})$. In $\mathrm{SO}(10)$, monopoles with masses $\sim 10^{4} \mathrm{GeV}$ could exist. On the other hand magnetic monopoles from GUTs with still larger groups, such as Kaluza-Klein theory or supersymmetry generally have higher mass, $m_{M} \gtrsim 10^{19} \mathrm{GeV}$. The magnetic charge of the GUT monopoles is predicted to be either 1 or 2 Dirac charges $\left(g_{D}\right)$.

Because of the extreme mass of the GUT monopoles, a total rethinking of search strategies was necessary. It is generally agreed that GUT monopoles can only have been created in the early $\left(\sim 10^{-35} \mathrm{sec}\right)$ hot Big Bang universe, possibly abundantly $\llbracket 4$. Thus the only GUT monopoles we can possibly find are primordial. 
Most of the experiments searching for them have been cosmic ray (or in-flight) experiments [0]. An alternate, possibly more efficient way is to search for magnetic monopoles trapped in matter. In the Big-Bang theory the GUT monopoles would be produced when the universe was $\sim 10^{-35}$ seconds old. Hydrogen and helium synthesis began when the universe was $\sim 1$ second old and ended when $\sim 10^{2}$ seconds old. Stars and galaxies are believed to form much later than that, when the universe is $\gtrsim 10^{12}$ seconds old. Heavy nuclei such as Fe and $\mathrm{Ni}$ are produced in stars by nuclear fusion. Magnetic monopoles, which might have been accreted in the stars, should mostly have survived the evolution process because of the exact conservation of magnetic charge. Annihilations are inhibited by even a small magnetic field and would not be expected to decrease the monopole density by a large factor. Eventually the monopoles may be recycled in supernova, along with the heavy nuclei, to form new stars and planets. Thus most of the magnetic monopoles may remain trapped in stars and other astronomical bodies, probably near the core. Presently the only macroscopic samples we have of material from inside such bodies are meteorites and meteoritic dust.

Monopoles can be bound to bulk ferromagnetic material by an image force. For iron (magnetite) the binding force near the boundary is $\sim 100 \mathrm{eV} / \mathrm{nm}(60 \mathrm{eV} / \mathrm{nm})$ [6], compared to the gravitational force $\sim 1 \mathrm{eV} / \mathrm{nm}$ on a monopole with mass $10^{17} \mathrm{GeV} / \mathrm{c}^{2}$ near the surface of the Earth. Thus a monopole with mass $\lesssim 10^{19} \mathrm{GeV} / \mathrm{c}^{2}$ would be bound. However if the sample containing the monopole is subjected to an acceleration, the monopole could be dislodged. Meteorites entering the Earth's atmosphere undergo accelerations of 100-1000 g. [7] The interiors of meteorites remain cold during their passage through the atmosphere. [8] Any monopoles they contain are likely to remain trapped in ferromagnetic grains in the interior. Thus the effective upper limit on the monopole masses that meteorites might contain is $\sim 10^{16}-10^{17} \mathrm{GeV} / \mathrm{c}^{2}$. Monopoles also lose $\sim 0.02 \mathrm{eV} / \mathrm{nm}$ in remagnetizing hard magnetic material such as magnetite or meteoritic iron, so that monopoles with mass $\lesssim 10^{14}$ $\mathrm{GeV} / \mathrm{c}^{2}$ would only be extracted by large sustained accelerations.

In general, primordial massive monopoles are unlikely to remain in material near the Earth's surface, because most of the Earth's material was at one time at a temperature well above the Curie point. This means that any primordial massive monopoles are likely to have fallen to the center. If there are magnetic monopoles with masses $\gtrsim 10^{16} \mathrm{GeV} / \mathrm{c}^{2}$ in rocks near the Earth's surface, they would have to be captured after the rocks formed and cooled down below the Curie temperature. However it is easy to show that the chance of a massive monopole being stopped near the Earth's surface is extremely low because the range of a monopole is typically much greater than the diameter of the Earth. [9]

Most meteors are fragments of small planets or asteroids. [8] This means that the meteorites falling on the Earth are samples from cores of small planets which might contain monopoles. Meteor ages are comparable to that of the Earth. Most have never been reheated above the Curie point and have a high content of iron. This will insure that monopoles will reman trapped in them via the monopole-image force if they have not been subjected to overly large accelerations as explained above.

In addition to meteorites themselves, sedimentary rocks and ocean floor sediments contain non-negligible amounts of meteoritic dust; this consists of very small grains of meteoritic material constantly falling down on the Earth. [10] Typically $\sim 20 \mu \mathrm{g}$ of meteoritic dust is found in $100 \mathrm{~g}$ of ordinary dust. [11] Also ferromanganese nodules found on the deep sea bed have especially large numbers of meteoritic spherules rich in Fe and Ni. [12] Another 
possibility is that monopoles could pass through part of the Sun, losing enough kinetic energy to be captured in solar orbits and eventually be stopped in the Earth. While it is not easy to estimate the possible monopole concentration due to these mechanisms, the rocks in the Earth's crust are also of some interest in trapped monopole searches considering their availability.

More than 20 years ago, Alvarez, Eberhard and collaborators [13] tested $\sim 20 \mathrm{~kg}$ of lunar rocks and other materials including $\sim 2 \mathrm{~kg}$ of meteorites. Another somewhat similar experiment was done by Ebisu and Watanabe. [14] They tested iron ore from one of the deep mines in Japan. The iron ore was heated above the Curie temperature [15] and they looked for monopoles which fell from the ore.

\section{THE MONOPOLE DETECTOR}

Physically the monopole detector is a cylindrically-shaped vacuum chamber as shown in Fig. 1. Its length is $1295 \mathrm{~mm}$ and its diameter is $457 \mathrm{~mm}$. The outer wall was made of mild steel to reduce the Earth's magnetic field. A mu-metal shield was placed just inside the outer wall for the same purpose. The center hole (warm bore) is $152.4 \mathrm{~mm}$ in diameter and served as the test path. To reduce thermal noise (so-called Johnson noise), this was made of G-10, an electrically non-conductive glass fiber/epoxy laminate. A liquid helium dewar, made of stainless steel, was placed concentrically. Welded stainless steel is an ideal material for constructing cryostats; however it is electrically conductive and, thus, a source of thermal noise. In this case the thermal noise was acceptable, since the dewar was at liquid helium temperature $\left(\sim 4.2^{\circ} \mathrm{K}\right)$ and samples with ambiguous monopole content could easily be passed through repeatedly.

The passage of a magnetic monopole (trapped in a sample) will cause a jump in the persistent current in the superconducting coil. This minute change of current in the coil is measured reliably with a SQUID. For a superconducting coil with $N$ turns and inductance $L$, the change in current is $\Delta i=4 \pi N g / L=2 \Delta i_{o}$, where $\Delta i_{o}$ is the current change for a change of one flux quantum of superconductivity, $\Phi_{o}=\hbar c / 2 e=2.07 \times 10^{-7} \mathrm{G}-\mathrm{cm}^{2}$. Here we assume that the magnetic charge of a monopole is equal to 1 minimal Dirac charge $\left(g_{D}=2 \Phi_{o}\right)$. A magnetic dipole will cause no net change of magnetic flux and thus no persistent current in the coil.

To reduce vibration the detector was suspended by 3 nylon lines attached to the 3 ends of a T-shaped aluminum bar. The bar was bolted to the ceiling via a vibration damper. Natural frequencies of the system were $\sim 1 \mathrm{~Hz}$ vertically and $\sim 0.5 \mathrm{~Hz}$ horizontally.

Most of the samples we tested had a fair amount of ferromagnetic material, and thus were strong magnetic dipoles in general. Thus, with some samples the SQUID output signal was changing even when the samples moved to the outermost positions. Sometimes these changes were indistinguishable from that expected from a magnetic monopole. This was mainly due the permanent dipole moment of the sample, but sometimes the dipole moment induced by the magnetic field outside the detector was as big as or bigger than the permanent dipole moment. The dipole signal was reduced significantly by placing the sample in a tight fitting mu-metal container when necessary.

The expected response of the detector to a magnetic monopole was simulated with a 
"pseudopole", which is a very long solenoid with a diameter of $9.5 \mathrm{~mm}$. The pseudopole was also used to verify that the SQUID measured the flux charge properly when it was subjected to a huge transient dipole field.

The signal from the SQUID controller was digitized by an analog-to-digital converter. Internal feedback was not used in the SQUID controller, so the electronics did not need to follow the large signals from dipole fields. The low pass filter in the SQUID controller was set to $1 \mathrm{~Hz}$, and we digitized the signal at a rate of $\sim 10$ per second. The digitized signal was recorded for 4 complete (up/down) cycles for each sample. One cycle took $\sim 40$ seconds, or $\sim 160$ seconds to collect one set of data. When the detector had settled down, about 4 hours after filling with liquid helium, the noise level was roughly one-third of the smallest Dirac charge $\left(=2 \Phi_{0}\right)$, as simulated by the pseudopole.

\section{DISCUSSION AND CONCLUSIONS}

We tested meteorites and other samples, amounting to more than 743 in number and $331 \mathrm{~kg}$ in total mass (Table I). This is about 10 times the total material tested by Alvarez, Eberhard et al. [13] We did not find a magnetic monopole. [16] With these results, we can conclude that the average overall ratio of the monopoles to nucleons in our sample is $n_{M} / n_{N}<1.2 \times 10^{-29}$.

If $m_{M} \gtrsim 10^{17} \mathrm{GeV}$, this experiment may not necessarily be a very good test of the monopole/nucleon ratio of the universe because the trapped monopoles could have been dislodged by accelerations which occurred during the meteorites entry into the atmosphere, their impact, or subsequent handling.

We wish to acknowledge the efforts of James Stone in early phases of the experiment. H.R. Gustafson provided considerable help and advice. We especially wish to thank J. Budai, E. Essene, D. Peacor, and Y. Zhang of the University of Michigan Department of Geological Sciences for loaning us many samples. We are grateful to the NASA Meteorite Working Group for the loan of 100 Antarctic meteorite samples. This work was supported by the National Science Foundation and grants from the Michigan Memorial Phoenix Project and the Office of the Vice President for Research. 


\section{REFERENCES}

[1] P.A.M. Dirac, Proc. Roy. Soc. London A133, 60 (1931).

[2] G. t'Hooft, Nucl. Phys. B79, 276 (1974).

[3] A. Polyakov, Pi'sma. Eksp. Teor. Fiz. 20, 430 (1974) [JETP Lett. 20, 194 (1974)].

[4] See, for example, the review by J. Preskill, Ann. Rev. Nucl. Part. Sci. 34, (1984).

[5] See, for example, the review by D.E. Groom, Phys. Rep. 140, 324 (1986).

[6] E. Goto, J. Phys. Soc. Japan 10, 1413 (1958); E. Goto, H.H. Kolm and K.W. Ford, Phys. Rev. 132, 387 (1963).

[7] V. Buchwald, Handbook of Iron Meteorites, Vol. 1 (Univ. of California Press, Berkeley, 1975).

[8] J.T. Wasson, Meteorites: Their Record of Early Solar-System History, (W.J. Freeman, NY, 1985).

[9] The energy loss of a bare monopole in matter is $0.1-1.0 \mathrm{GeV} / \mathrm{cm}$ for $v=10^{-3}-10^{-2} \mathrm{c}$. [S. Ahlen and K. Kinoshita, Phys. Rev. D26, 2347 (1982)] For a mass of $10^{17} \mathrm{GeV} / \mathrm{c}^{2}$ and a typical velocity of $10^{-3} \mathrm{c}$, it would take $10^{10}-10^{11} \mathrm{~cm}$ to stop the monopole, compared to the Earth's diameter of $1.28 \times 10^{9} \mathrm{~cm}$. If the monopole has a proton or aluminum nucleus attached, it might stop if the initial velocity is $\lesssim 10^{-3} \mathrm{c}$.

[10] P.W. Hodge, Interplanetary Dust, (Gordon and Breach Scientific Pub., NY, 1981).

[11] D.I. Olsson-Steel in Dust in the Universe, ed. by M.E. Bailey and D.A. Williams, p. 18 (Cambridge Univ. Press, Cambridge, 1987).

[12] D.E. Brownlee in Cosmic Dust, ed. by J.A.M. McDonnell, P. 295 (Wiley-Interscience, Chichester, 1978).

[13] L.W. Alvarez, P.H. Eberhard, R.R. Ross, and R. Watt, Science 167, 701 (1970); P.H. Eberhard, R.R. Ross, L.W. Alvarez, and R.D. Watt, Phys. Rev. D4, 3260 (1971); R.R. Ross, P.H. Eberhard, L.W. Alvarez, and R.D. Watt, Phys. Rev. D8, 698 (1973).

[14] T. Ebisu and T. Watanabe, Phys. Rev. D36, 3359 (1987). Note that this experiment is limited to a narrow range of monopole masses around $10^{15} \mathrm{GeV} / \mathrm{c}^{2}$ since it is assumed that the monopole mass is low enough to allow trapping in the iron (or magnetite) below the Curie point, but large enough for the monopole to fall out when raised above the Curie point.

[15] The Curie point of iron is $\approx 770^{\circ} \mathrm{C}$. That for magnetite is $\approx 585^{\circ} \mathrm{C}$. [Ref. 14].

[16] A more detailed description of the experiment is available in Searching for Magnetic Monopoles Trapped in Bulk Materials, H. Jeon, thesis (Univ. of Michigan Department of Physics, 1994). 


\section{TABLES}

TABLE I. Summary of Samples

\begin{tabular}{|c|c|c|c|c|}
\hline Samples $^{\mathrm{a}}$ & Mass & Number & Age & Notes \\
\hline Perry collection meteorites & $32.7 \mathrm{~kg}$ & $65 \mathrm{pcs}$ & $>10^{9}$ years & 2 \\
\hline Antarctic meteorites & $81.0 \mathrm{~kg}$ & 99 pcs & $>10^{9}$ years & 2 \\
\hline Ferromanganese nodules ${ }^{\mathrm{c}}$ & $5.0 \mathrm{~kg}$ & 31 pcs & $10^{2}-10^{7}$ years & 3 \\
\hline Iron ores-magnetite $^{\mathrm{d}}$ & $20.4 \mathrm{~kg}$ & 39 pcs & $\sim 10^{9}$ years & 4 \\
\hline Iron ores-hematite & $5.0 \mathrm{~kg}$ & $8 \mathrm{pcs}$ & $\sim 10^{9}$ years & 4 \\
\hline Blueschists ${ }^{\mathrm{e}}$ & $66.2 \mathrm{~kg}$ & 77 pcs & $\sim 10^{9}$ years & 5 \\
\hline Sedimentary rock cores ${ }^{\mathrm{f}}$ & $112.5 \mathrm{~kg}$ & $\approx 400 \mathrm{pcs}$ & $\sim 10^{9}$ years & 6 \\
\hline Kimberlites $^{\mathrm{g}}$ & $3.7 \mathrm{~kg}$ & 4 pcs & & 7 \\
\hline Chromates & $4.9 \mathrm{~kg}$ & 10 pcs & & \\
\hline Garnets $^{\text {h }}$ & $<1 \mathrm{gm}$ & $>10 \mathrm{pcs}$ & $\sim 10^{9}$ years & 8 \\
\hline Total & $331.4 \mathrm{~kg}$ & $>743$ pcs & & \\
\hline
\end{tabular}

${ }^{a}$ All the samples except for the Antarctic meteorites were borrowed from the University of Michigan Department of Geological Sciences. The Antarctic meteorites were borrowed from the NASA Meteorite Working Group.

${ }^{b}$ Perry collection meteorites were collected from all over the world. Antarctic meteorites were collected from Antarctica. Most of the meteorites we tested are chondrites.

${ }^{\mathrm{c}}$ Ferromanganese nodules were collected from South Pacific area (Clifferton-Clarion fracture zone). Depth of the area is $4.5-5.5 \mathrm{~km}$.

${ }^{\mathrm{d}}$ Magnetites were from mines all over the USA. They are ferrimagnetic. Hematites were mostly from mines in Michigan state and are anti-ferromagnetic.

e Blueschists are scaly sedimentary rocks which contain modest amounts of magnetite. They formed at depths of 20-45 km and have never experienced high temperature.

${ }^{\mathrm{f}}$ Sedimentary rock cores were drilled from $1.6 \mathrm{~km}$ depth, Branch Co., Michigan.

${ }^{g}$ Kimberlite are the base rocks in which diamonds are imbedded. They formed near the boundary between the earth's crust and mantle.

${ }^{\mathrm{h}}$ Garnets are also found in the blueschists. The garnets are formed at even higher pressure (thus depths) then the blueschists. 